\title{
Possibility on the Thermal Quench of the Superconducting Coil at the Loss-of-Coolant Accident
}

\author{
Kenzo IBANO and Yuichi OGAWA \\ Graduate School of Frontier Sciences, Kashiwa, Chiba 277-8568, Japan
}

(Received 25 December 2013 / Accepted 6 January 2014)

\begin{abstract}
Sequential phenomena of fusion reactor at anomaly events, e.g., sudden loss of coolant due to leaks or guillotine breaks at ex-vessel, should be carefully studied before the construction and operation of the fusion power demonstration reactor (DEMO). While a possibility of plasma termination due to impurity ejection from the wall by the loss of its cooling ability is usually considered, there might be a possibility of a plasma disruption induced by the thermal quench of the toroidal field (TF) coil due to the decrease of nuclear radiation shielding performance. Neutronics analyses taken for the Slim-CS DEMO designs with and without coolant water in the shield blanket showed that the nuclear heating rate at its TF coil increases more than 100 times by the coolant loss. Thermal calculation with the calculated heating rates indicated that the TF coil would reach the critical temperature within several seconds. Thus, a passive shutdown sequence other than the impurity from the wall sequence possibly occurs in case of the loss-of-coolant accident (LOCA) by a large penetration of neutrons onto the TF coils, a quench of coil current, and a termination of plasma confinement.
\end{abstract}

(c) 2014 The Japan Society of Plasma Science and Nuclear Fusion Research

Keywords: safety, neutronics, LOCA, superconducting coil, shield blanket

DOI: $10.1585 /$ pfr.9.1205006

Aiming to realization of fusion power, several concept designs of demonstration reactors (DEMO) have been studied all over the world [1-5]. Safety and soundness of these designs must be examined before starting the actual construction of fusion power plants. In case of ITER construction, its shutdown scenario at the abnormal events was analyzed by SAFALY [6] and AINA code [7]. Basically these codes couple a 0-D plasma dynamics model and a 1-D wall heat transfer model. Studies with these codes had shown the inherent safety of a fusion reactor, i.e., the ITER plasma will be passively shutdown because of impurity ejections from walls at anomaly incidents; e.g., a loss of coolant accident (LOCA) from components by leaks or guillotine breaks of an ex-vessel coolant pipe (ex-LOCA) or a sudden increase of plasma density accident.

In a normal circumstance, sensors should monitor conditions of these cooling lines and send a shutdown signal for its plasma control system. However, there will be certain delay times for the detection and for the disruption mitigation process. There might be a possibility that the shutdown sequence fails to terminate the plasma operation. In a safety point of view, sequential phenomena of the ex-LOCA while the plasma operation still continues due to some troubles should be carefully examined. It is preferred that the plasma operation passively shutdown without the devastating damages on the reactor components.

While the previous analyses have studied well about a passive shutdown by the impurity-from-the-wall sequence,

author'se-mail:kibano@ppl.k.u-tokyo.ac.jp a possibility of decreasing the nuclear radiation shielding performance due to the loss of coolant has not been discussed. It is predicted that the loss of cooling water, a common coolant as well as a good neutron reflector, strongly enlarges the neutron flux to the components, in particular to the toroidal field (TF) coil. If the neutrons flux to the superconducting coil increases significantly, the nuclear heating rate increases, the coil temperature increases, and a thermal quench of the TF coil may occur. The quench of the coil results a termination of plasma confinement, thus this sequence is another possibility of the passive shutdown of the plasma operation. This thermalquench-due-to-less-shielding sequence is strongly possible in the case of ex-LOCA of the shield blanket, where most of DEMO designs [1-5] assumes water-cooling, because temperature changes of these components merely influence the plasma facing surface, thus the impurity-from-the-wall sequence would not occur. In order to examine this prediction, neutronics and thermal calculations were taken for the ex-LOCA cases of Slim-CS [1] design, a water-cooled compact DEMO fusion reactor with reduced-size Centre Solenoid (CS). Neutronics calculations examine how the nuclear heating rate changes in case of the ex-LOCA at the shield blanket, and the thermal calculations for the TF coil examine how these changes influence the temperature of the coils.

Calculations on nuclear radiation transports and nuclear heating rates at the reactor components were taken for the in-board radial build design of the Slim-CS. The neutronics calculations were taken by THIDA [8] pack- 
Table 1 Slim-CS components materials and their radial thickness at the in-board side.

\begin{tabular}{|c|c|c|}
\hline & Materials & $\begin{array}{c}\text { Thickness } \\
\text { In-board side }\end{array}$ \\
\hline $\begin{array}{c}\text { FW/Breeding } \\
\text { blanket }\end{array}$ & $\mathrm{W} / \mathrm{F} 82 \mathrm{H} / \mathrm{LiTiO}_{3}+\mathrm{Be}_{12} \mathrm{Ti}$ & $30 \mathrm{~cm}$ \\
\hline Shield blanket & $\mathrm{F} 82 \mathrm{H}: \mathrm{H}_{2} \mathrm{O}=70: 30$ & $\sim 70 \mathrm{~cm}$ \\
\hline Coil case & $\mathrm{SUS} 316 \mathrm{LN}$ & $7 \mathrm{~cm}$ \\
\hline TF coil & $\begin{array}{c}\mathrm{Nb}_{3} \mathrm{Al}, \mathrm{Copper}, \\
\mathrm{SUS} 316 \mathrm{LN}, \mathrm{JJ} 1\end{array}$ & $110 \mathrm{~cm}$ \\
\hline
\end{tabular}

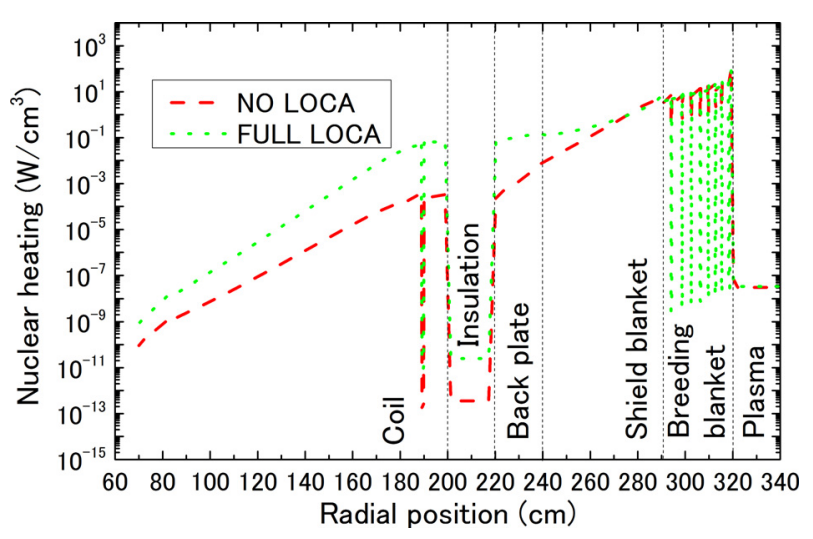

Fig. 1 Calculated radial distribution of nuclear heating rate with and without cooling water for Slim-CS in-board side.

age based on the 1-D transport simulation code ANISN [9] with the FENDL2.0 cross section library. Brief description of the Slim-CS design used in the calculation was summarized in Table 1. In the calculation model, a first TF superconductor strand on the plasma side was modeled in detail. The detail model consists of insulators, structures, metal tapes, superconductor materials, and He coolants. Rear strands were modeled as mixed materials of them. Calculations were repeated for models with different amounts of residual coolant in the shield.

Radial profile of calculated nuclear heating rate at the Slim-CS was plotted in Fig. 1. Result of a calculation on an ex-LOCA case where coolant water in the shield blanket is absent was also plotted in Fig. 1. Calculated neutron and radiation flux at the TF coil and nuclear heating rate at the first strand of coil were summarized as a function of the residual coolant amount in Fig. 2. Transient temperature change of the TF coil was analyzed using calculated nuclear heating rate as the heat source. Here, a special attention was paid for the heat capacity which value is extremely low $(\sim 1 \mathrm{~J} / \mathrm{kg} \mathrm{K})$ at the ultimate low temperature. Calculated temperature change as a function of the residual coolant amount was summarized in Fig. 3.

As shown in Fig. 1 and Fig. 2, the nuclear heating rate significantly increases as the residual coolant amount decreases. For the Slim-CS case, its nuclear heating

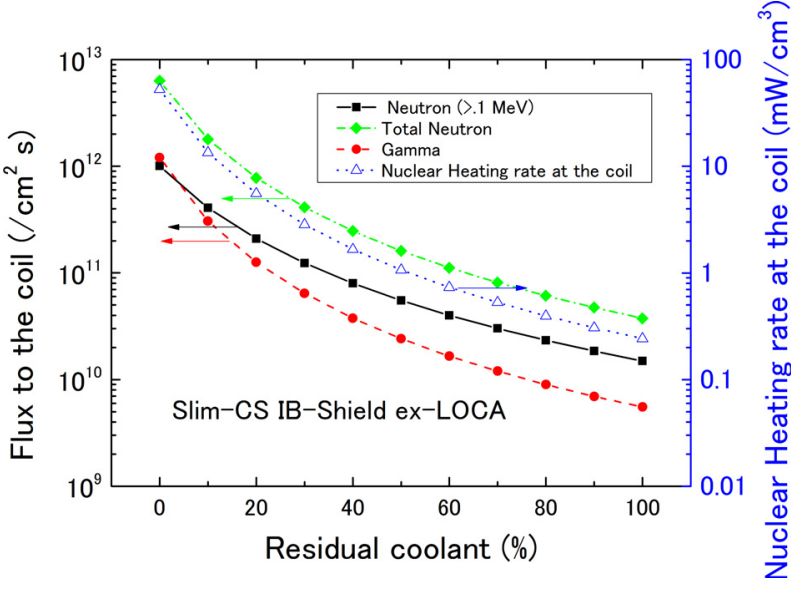

Fig. 2 Neutron and gamma ray radiation flux as well as nuclear heating rate at the TF coil as a function of residual coolant in the in-board shield blanket. Calculation was taken by THIDA package with FENDL2.0 cross-section library.

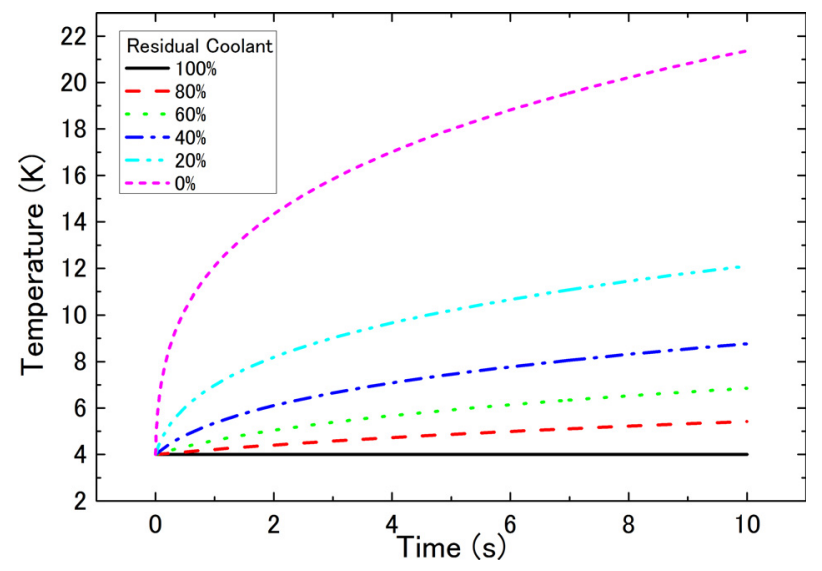

Fig. 3 Change of coil temperature after the accidentally loss of coolant at the shield blanket as a function of residual coolant amount.

rates at the $\mathrm{TF}$ coil can increase from $0.2 \mathrm{~mW} / \mathrm{cm}^{3}$ to $52.5 \mathrm{~mW} / \mathrm{cm}^{3}$ at a maximum by the ex-LOCA on the shield blanket. This increasing volumetric heating rate results a sudden temperature increase in the TF coil because of the extremely low heat capacity as shown in Fig. 3. Assuming the coolant completely disappears suddenly at $t=0$, thermal calculations indicate that the TF coil temperature would reach the critical temperature within several seconds as shown in Fig. 3. Thus, the ex-LOCA of the radiation shield would result the thermal quench of the TF coil and the termination of the plasma operation. Although further detailed analyses should be taken, a possibility of a fusion reactor passive shutdown mechanism for the ex-LOCA by the thermal-quench-due-to-less-shielding sequence has been indicated with this study.

A concern indicated with this study is a possibility of a "non-local" quench. The increasing nuclear heating 
rate due to the ex-LOCA would happen simultaneously at a wide area of the TF coil. Thus, the quench would also be caused at the wide area. While an effect of thermal quench caused by a local heat spot has been well studied, uncertainties still remain on an effect of the non-local and widearea quench. Thus, thermal and thermo-mechanical analyses on the TF coil after the quench and a pressure analysis of the coil coolant should be taken for this non-local and wide-area quench.

Nuclear transport analysis showed that decreasing shielding performance by the loss of its cooling water at the shield blanket would results increasing nuclear heating rate at the TF coil. Results indicated that the TF coil quench due to this increasing nuclear heat would happen within several seconds. Thus, even in the case of ex-LOCA at the cooling line of the shield, when the impurity-fromthe-wall sequence cannot terminate the plasma, the plasma operation would be passively terminated by the thermal- quench-due-to-less-shielding sequence. Uncertainties still remain for degrees of damage to the components by the accident.

Countless fruitful discussions were taken with Prof. Matsuda at Kyoto University from the beginning of this study. Advice and design information of Slim-CS were given by the JAEA reactor system study team.

[1] K. Tobita et al., Nucl. Fusion 47, 892 (2007).

[2] R. Hiwatari et al., Nucl. Fusion 45, 96 (2005).

[3] K. Ibano et al., Fusion Eng. Des. 86, 2779 (2011).

[4] F. Nahamabadi and the ARIES team, Fusion Eng. Des. 65, 143 (2003)

[5] D. Maisonneir et al., Fusion Eng. Des. 75-79, 1173 (2005).

[6] T. Honda et al., J. Fusion Energy 16, 175 (1997).

[7] J.C. Rivas and J. Dies, Fusion Eng. Des. 88, 2709 (2013).

[8] S. Mori et al., JAERI 1301 (1986).

[9] W.W. Engle Jr., Oak Ridge Report K-1693 (1967). 